\title{
Influence of Frying Methods on Quality Characteristics and Volatile Flavor Compounds of Giant Salamander (Andrias davidianus) Meatballs
}

\author{
Wengang Jin ${ }^{(D)},{ }^{1}$ Jinjin Pei, ${ }^{1}$ Xiaohua Chen, ${ }^{1}$ Jingzhang Geng, ${ }^{1}$ Dejing Chen ${ }^{D}{ }^{1}$ \\ and Ruichang Gao ${ }^{1,2}$ \\ ${ }^{1}$ Key Laboratory of Bio-Resources of Shaanxi Province, School of Biological Science and Engineering, \\ Shaanxi University of Technology, Hanzhong 723001, China \\ ${ }^{2}$ School of Food and Biological Engineering, Jiangsu University, Zhenjiang 212013, China
}

Correspondence should be addressed to Wengang Jin; jinwengang@nwafu.edu.cn and Ruichang Gao; xiyuan2008@ujs.edu.cn Received 7 July 2021; Accepted 5 August 2021; Published 27 August 2021

Academic Editor: Alessandra Del Caro

Copyright (c) 2021 Wengang Jin et al. This is an open access article distributed under the Creative Commons Attribution License, which permits unrestricted use, distribution, and reproduction in any medium, provided the original work is properly cited.

Effects of deep fat frying and hot air frying on texture, color difference, sensory score, yield, fat content, and volatile flavor compounds of giant salamander meatballs before and after frying were investigated. The results showed that, compared with the deep fat frying group, hot air-fried giant salamander meatballs had higher hardness, elasticity, and $L^{*}(p<0.05)$, but lower $a^{*}, b^{*}$ value, fat content, and yield $(p<0.05)$. There was little distinction in sensory score, cohesiveness, and chewiness between the two frying methods $(p>0.05)$. Gas chromatography ion migration chromatography (GC-IMS) was used for flavor compound analysis, and 50 flavor compounds were analyzed, containing 22 aldehydes, 11 ketones, 6 olefins, 4 acids, 3 esters, 3 alcohols, and 1 phenol. Compared with the samples before frying, the relative contents of aldehydes and ketones of fried giant salamander meatballs increased significantly, while the relative contents of esters and alkenes decreased significantly. Principal component analysis showed that the GC-IMS spectra of volatile flavor compounds before and after deep fat frying and hot air frying varied greatly, and the cumulative contribution rate of the two principal components reached $86.1 \%$, indicating that the GC-IMS technology might be used to distinguish giant salamander meatballs before and after frying, or with different frying methods. These results may offer a note for development and quality control of the precooked giant salamander meatballs in the future.

\section{Introduction}

Giant salamander (Andrias davidianus), also known as "babyfish," is the largest amphibian in the world [1]. This species enjoys high edible and medicinal value, because its skin, skin secretion, muscle, blood, and bone contain many bio-active substances [2]. At present, artificial culture of Andrias davidianus has been industrialized in Hanzhong, Zhangjiajie, Henan, Luoyang, Guizhou, Sichuan, and Chongqing (China) $[3,4]$. Owing to its growing quantity and diminishing cost, a lot of work have been done to transform cultured Andrias davidianus to high-valued commodities [4]. In recent years, there are more and more reports about the nutritional evaluation of giant salamander meat [5], cutting and preservation [6], and bioactive ingredients [7-10]. Processing plants also develop commercial Andrias davidianus products such as small-packaged cut meat, meatballs, collagen, and oil [11], which greatly promote the transformation and development of giant salamander industry.

Meatballs are a kind of traditional minced meat product widely processed and consumed, which become more and more popular for their delicious taste, balanced nutrients, and convenience [12-14]. Frying is one of the common cooking methods for meatballs. Ateba and Mittal [15] investigated the process of coat evolution and attributes of meatballs suffering from frying. Wang et al. [16] found that ultrasonic-aided frying could improve 
some physicochemical indexes of beefballs. Zhang et al. [17] further proved that ultrasonic-aided frying could improve the flavor profiles of meatballs. After frying, meatballs are deodorized and endowed with aroma, maintaining a better appearance by denaturing meat protein and solidifying at high temperature $[14,15,18]$. However, high calorie and oil content of fried food make consumers anxious about chronic cardiovascular disease [18-20]. How to balance the needs of color, fragrance, taste, and nutrition of fried food is a hot issue for modern food industry.

Hot air frying (HAF) is a novel emerging food frying method in recent years, which directly smears a small amount of edible oil on the surface of raw materials and then uses circulating hot air to heat and cook [19-21]. Compared with the traditional frying process, HAF food gives the appearance and texture of food fried to a certain extent and also has the advantages of less frying oil consumption and lower oil content [20-23], which is considered as one of the novel frying technologies for replacement of the traditional frying process. Ghaitalampour et al. [24, 25] compared the donut quality differences of traditional frying and hot air frying and established a heat and mass conduction model of hot air-fried donut, which might be employed to predict the changes of water and heat during frying. Gouyo et al. [26] found that traditional French fries are more brittle than hot air-fried through puncture test and sensory evaluation. Apart from these, the nutritional and quality characteristics of air-fried Coregonus peled meat [19], hairtail [22], and Penaeus vannamei [23] also confirmed the potential advantages of air-fried products over traditional fried ones.

With the industrialization of segmentation and processing of giant salamander, quick-frozen meat slices and meatballs of Andrias davidianus have become important commodities [11]. Fried meatball could add giant salamander value and commodity variety, but information about quality traits of Andrias davidianus meatball after frying was scarce. The target of present investigation was to explore the effects of hot air frying and traditional deep fat frying upon quality characteristics and volatile compounds of giant salamander meatballs (GSMs), providing a basis for ready-to-eat meatballs of giant salamander in future.

\section{Materials and Methods}

2.1. Raw Materials and Reagents. Fresh giant salamander meatballs (each $1 \mathrm{~kg}$ of giant salamander meat, containing $20 \mathrm{~g}$ of pig backfat, $100 \mathrm{~g}$ of starch, $15 \mathrm{~g}$ salt, $5 \mathrm{~g}$ of granulated sugar, $8 \mathrm{~g}$ of egg white, $5 \mathrm{~g}$ of chopped green onion, $5 \mathrm{~g}$ chopped ginger, $2 \mathrm{~g}$ of tripolyphosphate, and $100 \mathrm{~g}$ of ice water) were purchased from meatball processing lines of Longtoushan Aquaculture Development Co., Ltd.(Hanzhong, China). Once transported to the laboratory, they were vacuum-packaged and put at $4^{\circ} \mathrm{C}$ in a refrigeration device (ZX-CXG-600, Zhixin Instrument Co. Ltd., Shanghai, China). Edible rapeseed oil was bought from Yihai Kerry Food Co., Ltd (Xianyang, China). 6 standard ketones of analytical grade were bought from Guoyao Chemical Reagent Co., Ltd (Shanghai, China).
2.2. Manufacture of Fried Andrias davidianus Meatballs. Chilled Andrias davidianus meatballs with relatively uniform size (approximately $23.24 \mathrm{~kg} \pm 15 \mathrm{~g}$ ) were screened out. These meatballs were stored at room temperature and divided into two frying groups, namely, deep fat frying (DFF) and hot air frying (HAF). For DFF meatballs, 50 fresh meatballs were dipped and fried at $180^{\circ} \mathrm{C}$ for $10 \mathrm{~min}$ with rapeseed fat in a fat bath device (SHJ-1, Changzhou RRT Experimental Facility Co., Ltd., China). For HAF meatballs, 50 fresh meatballs were smeared with rapeseed oil, and then they were put in a hot air frying device (SB-021, Cixi Shanben Electric Appliance Co., Ltd, China) fried at $180^{\circ} \mathrm{C}$ for $10 \mathrm{~min}$. Fresh meatballs before frying (BF) were used as control. The quality parameters of fried meatballs were immediately evaluated after cooling to room temperature.

2.3. Yield Estimation. The yield of fried meatballs was estimated according to modified methods [27, 28]. Briefly, 5 meatball samples were weighed before and after frying, respectively. The yield was counted by the weight difference before and after frying, according to the following equation:

$$
\text { yield }(\mathrm{g} / 100 \mathrm{~g})=\frac{\text { sample weight after frying }(\mathrm{g})}{\text { sample weight before frying }(\mathrm{g})} \times 100 \text {. }
$$

2.4. Determination of Fat Content. The fat of meatballs was extracted by Soxhlet method as described previously [29]. Briefly, $50 \mathrm{~g}$ grounded meatball was extracted with $150 \mathrm{~mL}$ petroleum ether at room temperature with steady agitating for $10 \mathrm{~h}$. The broth was executed for centrifugation $(8,000 \mathrm{~g}$, $20 \mathrm{~min}$ ), and the liquid supernatant was loaded to a reduced pressure concentrator for solvent evacuation. The weight difference before and after extraction was used to assess fat content.

2.5. Texture Profile Analysis. The texture profile analysis of 10 meatballs before and after frying (DFF and HAF) was performed in a CT3 Texture Analyzer (Brookfield Co., Ltd. USA), with an adjusted procedure of Wang et al. [16]. Fried meatball samples were cut uniformly prior to analysis. Instrument parameters were set as follows: TPA mode, TA5 probe, pre-test speed: $2.0 \mathrm{~mm} / \mathrm{s}$, test speed: $2.0 \mathrm{~mm} / \mathrm{s}$; posttest speed: $2.0 \mathrm{~mm} / \mathrm{s}$; compression depth: $10 \mathrm{~mm}$; induction load: $5.0 \mathrm{~g}$. At the end of the test, hardness, elasticity, cohesiveness, and chewiness were selected in the parameter interface to output the results. 5 meatball samples of each fried group were tested in parallel, and the average value was calculated.

2.6. Color Value Measurement. The surface color of meatballs by the CIELAB color system was assayed on a digital colorimeter (CR-400, Konica Minolta Co., Ltd., Japan), according to an adjusted method of Jin et al. [28]. Before measurement, the colorimeter was calibrated with a black and wight plate; then the $L^{*}$ (lightness), $a^{*}$ (redness), and $b^{*}$ 
(yellowness) of 5 samples for each frying group were read, respectively. 5 surface points of each meatball were read to acquire color values as mean.

2.7. Sensory Assessment. Sensory assessment of the fried meatball samples was assessed by the Hedonic test, according to a modified approach described previously [27]. Ten panelists with no rhinitis and smoking ( 5 males and 5 females, age of 22-32) were made of postgraduates from present laboratory. Three training sessions were held to familiarize the panelists with the aim, detailed experimental steps, and requirements of sensory assessment before serving meatballs of the two frying group. Cold meatballs (5 for each frying group) were sliced before serving in a random order. Each assessor provided their scores through a 9-point hedonic scale: $1.0-3.0$ was deemed as not acceptable, 4.0-5.0 fairly acceptable, 6.0-7.0 good (acceptable), and 8.0-9.0 very good [28].

2.8. Analysis of Volatile Flavor Compounds. Volatile flavor compounds of meatball samples before and after frying were detected on a HS-GC-IMS instrument (FlavourSpec ${ }^{\circledR}$, Germany), according to a modified method previously [11]. Briefly, $2.0 \mathrm{~g}$ of grounded meatball sample was put into the $20 \mathrm{~mL}$ headspace bottles and implanted $(200 \mu \mathrm{L})$ by a high temperature injector $\left(85^{\circ} \mathrm{C}\right)$ when keeping at $60^{\circ} \mathrm{C}$ for $15 \mathrm{~min}$. The unbranched procedure was adopted. The gas chromatographic prefractionation was done at $60^{\circ} \mathrm{C}$ on a MXT-5 column $(15 \mathrm{~m} \times 0.53 \mathrm{~mm})$. The $99.99 \%$ nitrogen was as a vehicle air at a programmed speed as follows: $2 \mathrm{~mL} / \mathrm{min}$ for $2 \mathrm{~min}, 30 \mathrm{~mL} / \mathrm{min}$ for $8 \mathrm{~min}, 100 \mathrm{~mL} / \mathrm{min}$ for $10 \mathrm{~min}$, and $150 \mathrm{~mL} / \mathrm{min}$ for $5 \mathrm{~min}$. Then, the mixture gas was ionized in the IMS ionization cell. To avert cross pollution, the injector was compulsorily planed $30 \mathrm{~s}$ before single assay and $5 \mathrm{~min}$ after single assay. The $n$-ketones C4-C9 were as foreign standards to estimate the retention index (RI) of single volatile chemical. Via collations of RI and the drift time (DT) through the instrumental database (FlavourSpec ${ }^{\circledR}$, Germany), the volatile flavor substances were characterized through comparing IMS drift time and retention index with those of the standard chemicals. The signal intensity denotes the height or the peak area.

2.9. Statistical Procedure. The data were expressed as mean \pm standard deviation $(n \geq 3)$, and $t$-test was used for significance analysis $(p<0.05)$. Figures were plotted through Origin Pro 2018 (OriginLab Co., USA). The instrumental analysis included GC $\times$ IMS Library Search, LAV (Laboratory Analytical Viewer), and gallery plot. According to all volatile compounds identified between samples, a plug-in PCA score and biplot diagram (through normalization and eigenvectors) by the HS-GC-IMS instrument were also acquired [30].

\section{Results and Discussion}

3.1. Effect of Deep Fat Frying and Hot Air Frying on Quality Traits of Meatballs. This investigation fried giant salamander meatballs both by deep fat frying (DFF) and by hot air frying (HAF), and the appearance photo of the fried meatballs is shown in Figure 1.

Several quality parameters (yield, texture, fat content, color values, and sensory scores) of giant salamander meatballs after deep fat frying and hot air frying were also evaluated and are summarized in Table 1. As can been seen, the hardness and elasticity for HAF were relatively higher $(p<0.05)$, whereas the cohesiveness, chewiness, and sensory score for DFF and HAF were comparable. The $L^{*}$ value of HAF meatballs was greater than that of DFF meatballs $(p<0.05)$, but the $a^{*}$ and $b^{*}$ values were lower than those of DFF group $(p<0.05)$. The lower $L^{*}$ value and higher $a^{*}$ and $b^{*}$ values for DFF meatballs were mainly because of more oil absorbed and severer Maillard reaction during frying, whereas HAF meatballs were secondary [21, 24]. Zhao et al. [19] also found that the hardness, chewiness, and $L^{*}$ value of air-fried Coregonus peled meat were obviously greater than those of traditional fried fish, which was somewhat consistent with the above results of this study. However, the $a^{*}$ and $b^{*}$ values are different from the present results, probably due to the differences in raw materials, the type of frying fat, and the test conditions [21]. The sensory score of DFF giant salamander meatballs was higher than that of HAF, but the difference was not significant. The fat content and yield of DFF giant salamander meatballs were obviously greater than those of HAF $(p<0.05)$, because the deep fat frying process of giant salamander meatballs not only caused water loss, but also absorbed part of frying fat, resulting in the final fat content and yield of fried giant salamander meatballs higher than those of hot air-fried group [24-26]. A similar result of fat content and cook loss of fish by deep fat frying and hot air frying was also reported previously [19].

3.2. Volatile Flavor Compounds of Meatballs Based on HS-GCIMS. The volatile flavor substances of foods are traditionally detected by GC-MS, yet this instrument needs complicated procedures and long detection time, thus being of limited applicability to the fast analysis and isobaric compounds analysis [31, 32]. Oppositely, headspace-gas chromatography ion mobility spectrometry (HS-GC-IMS) is becoming a novel gas-phase prefractionation and assay tool for flavor compounds analysis with advantage of comparatively high sensitivity and simple sample preparation [30, 31]. The present study analyzed volatile flavor compounds of giant salamander meatballs before and after frying with a HS-GCIMS method, and the three- and two-dimensional views of spectrum are shown in Figure 2.

Figure 2(a) depicts 3D spectrum of the volatile flavor compounds of giant salamander meatballs before and after frying, obtained by reporter plug-in program of LAV analysis software of HS-GC-IMS instrument used. The vertical axis denotes the retention time, and the horizontal axis denotes the drift time. The red vertical line denotes the signal strength of the ion peak, single spot on both sides of the ion peak denotes a volatile component, and the shade of color marks the level of the content. A compound may have one, two, or more spots (denoting monomer, dimer, or 


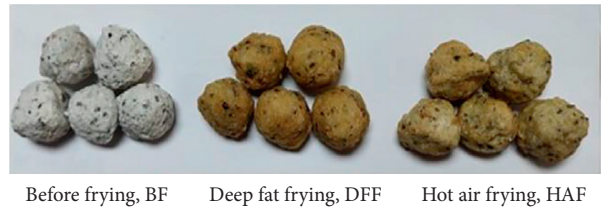

Figure 1: Appearance photo of giant salamander meatballs by deep fat frying and hot air frying.

TABLE 1: Texture, color, sensory scores, fat content, and yield of giant salamander meatballs after two frying methods.

\begin{tabular}{lcc}
\hline Quality index & & Group \\
& Deep fat frying (DFF) & Hot air frying (HAF) \\
\hline Hardness (g) & $486.33 \pm 33.93^{\mathrm{b}}$ & $516.08 \pm 18.82^{\mathrm{a}}$ \\
Cohesiveness & $0.51 \pm 0.08^{\mathrm{a}}$ & $0.48 \pm 0.12^{\mathrm{a}}$ \\
Elasticity & $8.08 \pm 0.12^{\mathrm{b}}$ & $8.43 \pm 0.37^{\mathrm{a}}$ \\
Chewiness & $20.77 \pm 5.01^{\mathrm{a}}$ & $28.00 \pm 4.70^{\mathrm{a}}$ \\
$L^{*}$ & $13.41 \pm 0.96^{\mathrm{b}}$ & $17.98 \pm 0.34^{\mathrm{a}}$ \\
$a^{*}$ & $4.74 \pm 0.15^{\mathrm{a}}$ & $4.17 \pm 0.09^{\mathrm{b}}$ \\
$b^{*}$ & $14.84 \pm 1.78^{\mathrm{a}}$ & $11.35 \pm 0.28^{\mathrm{b}}$ \\
Sensory score & $7.52 \pm 0.16^{\mathrm{a}}$ & $7.30 \pm 0.33^{\mathrm{a}}$ \\
Fat content (\%) & $22.64 \pm 0.20^{\mathrm{a}}$ & $13.23 \pm 0.12^{\mathrm{b}}$ \\
Yield (\%) & $72.99 \pm 2.04^{\mathrm{a}}$ & $65.16 \pm 3.25^{\mathrm{b}}$ \\
\hline
\end{tabular}

Different lowercase letters in the same row mark significant differences $(p<0.05)$.

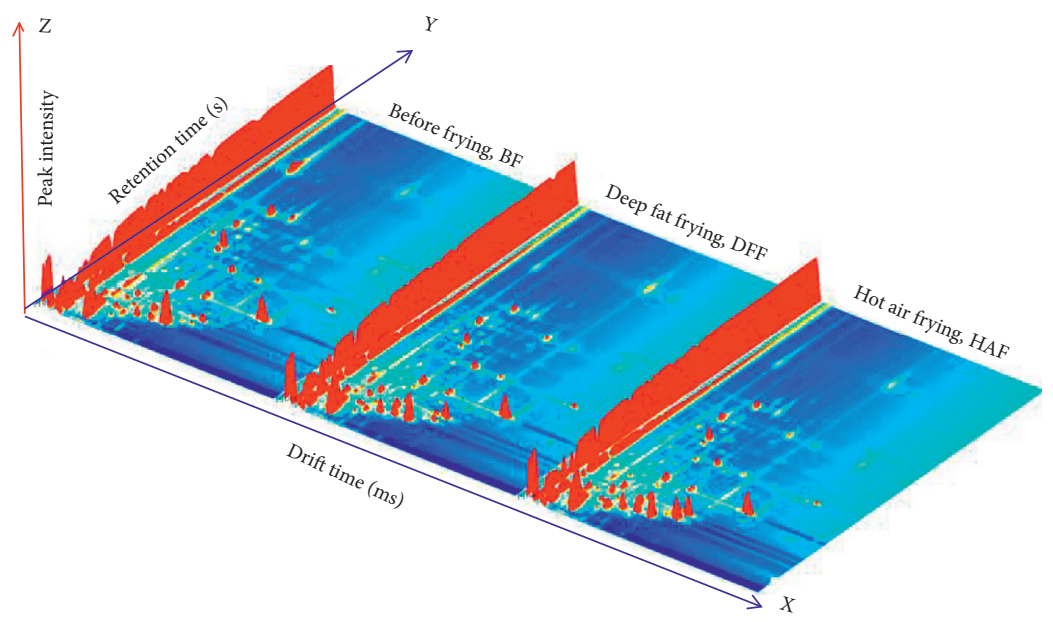

(a)

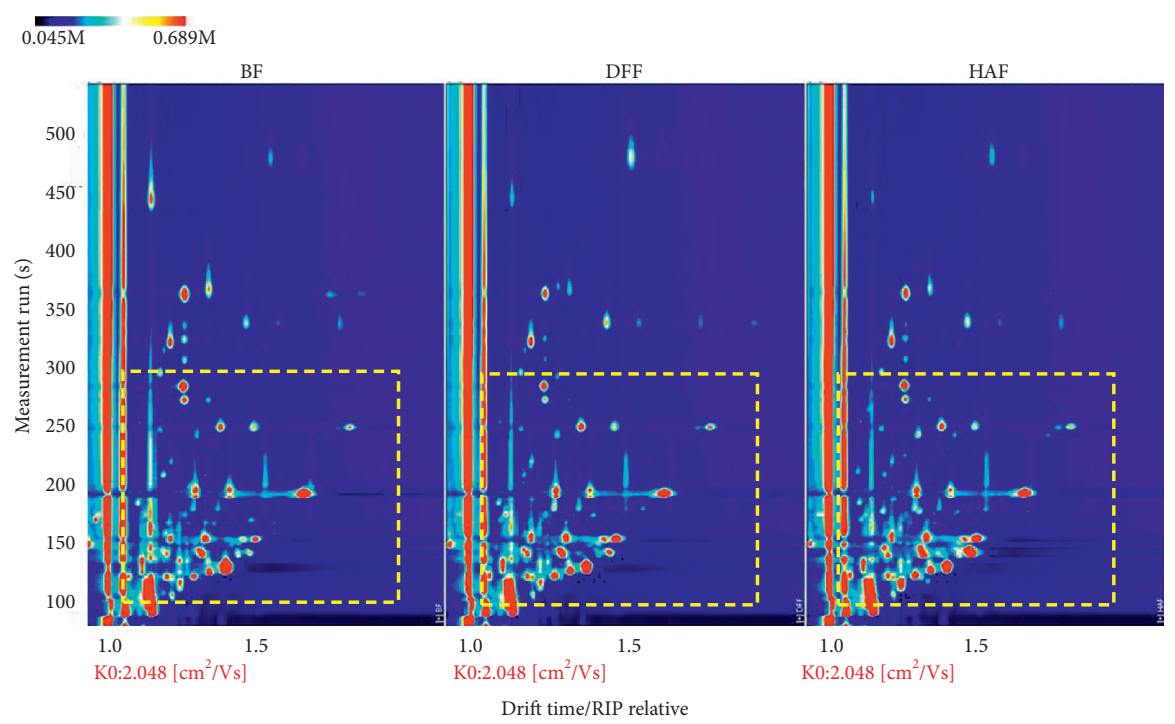

(b)

FiguRE 2: GC-IMS spectra of giant salamander meatball before and after frying. (a) Three-dimensional view; (b) two-dimensional view. 


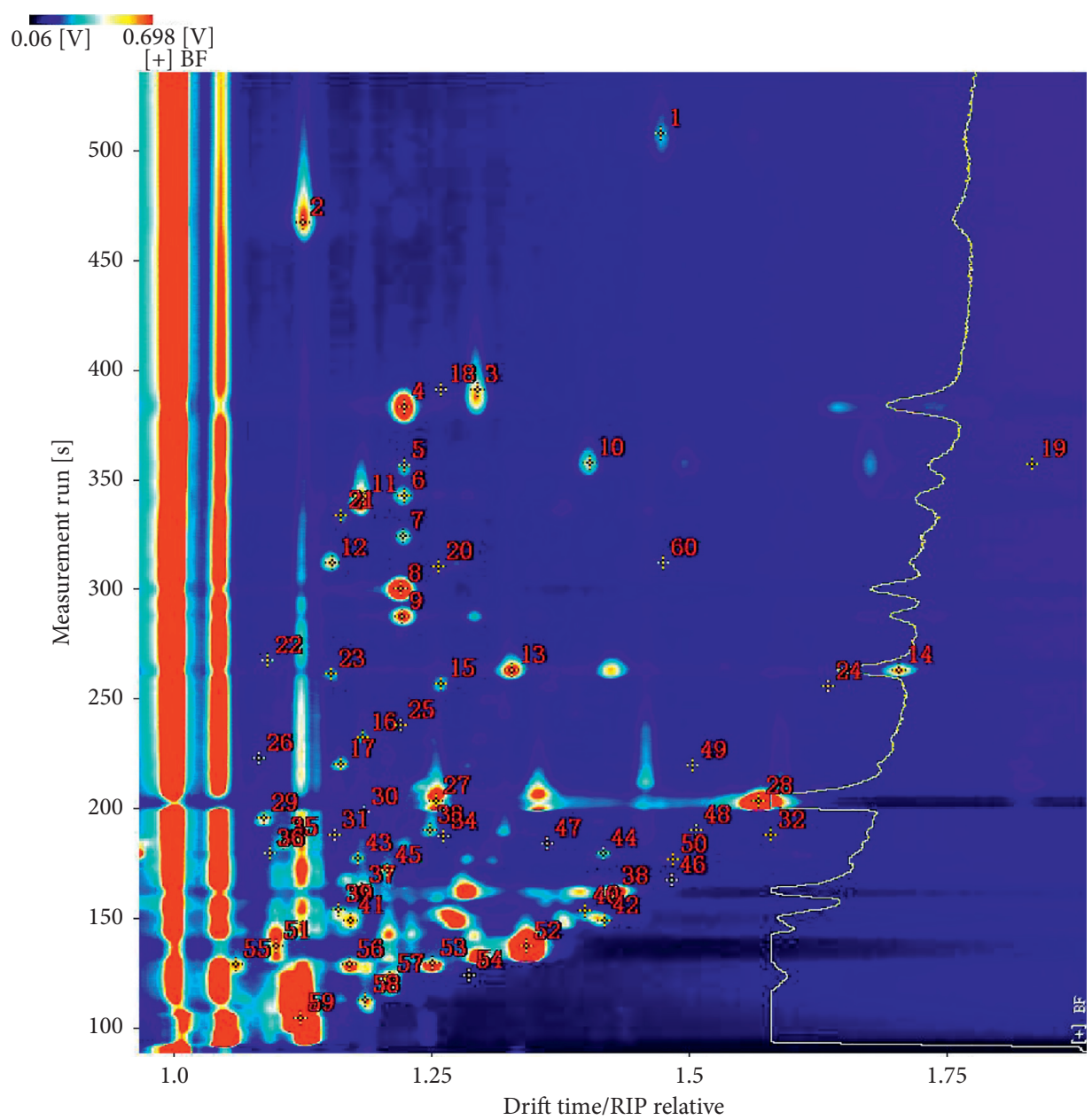

FIGURE 3: IMS qualitative analysis of volatile flavor substances of giant salamander meatball before frying.

trimer), influenced by the content and attributes of the specific volatile substance $[11,30,33]$. Figure 2(a) (left to right) shows the volatile compounds of meatball were $\mathrm{BF}$, $\mathrm{DFF}$, and HAF, respectively. Given the appearance of the spectrum, it is hard to directly distinguish the $3 \mathrm{D}$ spectrum of the samples before and after frying with the naked eye. The 3D spectrum was projected to a 2D plane view (Figure 2(b)), so that the difference of volatile flavor compounds before and after frying can be directly compared. The three groups of samples showed different HS-GC-IMS characteristic flavor profiles, and the concentrations of flavor substances in different fried samples increased or decreased and relatively obvious differences are shown in the yellow dotted box (Figure 2(b)). The characteristic spectrum of samples with different frying methods exhibited certain differences, probably due to the variation in heat and mass transfer, crust formation, nonenzymatic browning extent, and other factors during frying $[15,19,20,24]$.

3.3. Qualitative Analysis of Volatile Flavor Components. After comparing the retention time and migration time of characteristic flavor substances, external standard ketone C4-C9 was employed as a consult to estimate the retention index of each volatile substance and conduct qualitative analysis of the volatile substance through matching with GC-IMS database. Figure 3 shows the IMS qualitative analysis of volatile flavor compounds of giant salamander meatball (taking meatballs before frying as example). The marked number shown in Figure 3 represents a volatile flavor substance that was qualitatively analyzed. By the NIST gas retention index database and IMS database built in the instrumental software, a total of 50 kinds of monomers and dimers of volatile flavor substances were qualitatively identified, including 22 aldehydes, 11 ketones, 6 alkenes, 4 acids, 3 esters, 3 alcohols, and 1 phenol. The chemical formula, CAS number, retention time, retention index, drift time, and relative content of the identified monomers and dimers are the same, and the results are shown in Table 2.

3.4. Volatile Components Fingerprint of Giant Salamander Meatballs before and after Frying. To directly compare the effect of different frying processes on volatile flavor substances, giant salamander meatballs were tested in 3 parallelly to obtain signal peaks of GS-IMS under a 2D spectrum, and the volatile flavor fingerprints of meatballs before and after two frying processes are generated in Figure 4. 
TABLE 2: Qualitative analysis of volatile flavor substances of giant salamander meatball before and after frying.

\begin{tabular}{|c|c|c|c|c|c|c|c|c|c|c|}
\hline \multirow{2}{*}{ Number } & \multirow{2}{*}{ Volatile flavor compounds } & \multirow{2}{*}{ CAS } & \multirow{2}{*}{ MF } & \multirow{2}{*}{ MW } & \multirow{2}{*}{ RI } & \multirow{2}{*}{ RT (s) } & \multirow{2}{*}{ DT (ms) } & \multicolumn{3}{|c|}{ Relative content (\%) } \\
\hline & & & & & & & & $\mathrm{BF}$ & DFF & HAF \\
\hline 1 & Nonanal & C124196 & $\mathrm{C}_{9} \mathrm{H}_{18} \mathrm{O}$ & 142.2 & 1109 & 507.757 & 1.47366 & 0.88 & 2.29 & 1.24 \\
\hline 2 & 4-Methylphenol & C106445 & $\mathrm{C}_{7} \mathrm{H}_{8} \mathrm{O}$ & 108.1 & 1080.8 & 467.106 & 1.12665 & 4.42 & 1.78 & 1.00 \\
\hline 3 & Hexanoic acid & $\mathrm{C} 142621$ & $\mathrm{C}_{6} \mathrm{H}_{12} \mathrm{O}_{2}$ & 116.2 & 1028 & 391.156 & 1.29602 & 2.71 & 1.38 & 1.42 \\
\hline 4 & Limonene & C138863 & $\mathrm{C}_{10} \mathrm{H}_{16}$ & 136.2 & 1022.6 & 383.477 & 1.22572 & 5.26 & 3.23 & 3.31 \\
\hline 5 & Delta-carene & C29050337 & $\mathrm{C}_{10} \mathrm{H}_{16}$ & 136.2 & 1003.8 & 356.432 & 1.22572 & 0.45 & 0.23 & 0.23 \\
\hline 6 & Beta-pyronene & C514965 & $\mathrm{C}_{10} \mathrm{H}_{16}$ & 136.2 & 992.8 & 342.743 & 1.22572 & 0.95 & 0.52 & 0.57 \\
\hline 7 & Beta-pinene & C127913 & $\mathrm{C}_{10} \mathrm{H}_{16}$ & 136.2 & 970.9 & 324.046 & 1.22422 & 0.74 & 0.37 & 0.46 \\
\hline 8 & Alpha-fenchene & C471841 & $\mathrm{C}_{10} \mathrm{H}_{16}$ & 136.2 & 943.1 & 300.341 & 1.22123 & 6.52 & 3.87 & 4.30 \\
\hline 9 & Alpha-pinene & C80568 & $\mathrm{C}_{10} \mathrm{H}_{16}$ & 136.2 & 928.2 & 287.654 & 1.22273 & 2.11 & 1.19 & 1.44 \\
\hline 10 & Octanal-M & C124130 & $\mathrm{C}_{8} \mathrm{H}_{16} \mathrm{O}$ & 128.2 & 1004.7 & 357.694 & 1.405 & 0.95 & 1.76 & 1.40 \\
\hline 11 & Methyl-5-hepten-2-one & C110930 & $\mathrm{C}_{8} \mathrm{H}_{14} \mathrm{O}$ & 126.2 & 990.7 & 340.973 & 1.18528 & 3.04 & 3.66 & 3.53 \\
\hline 12 & Benzaldehyde-M & C100527 & $\mathrm{C}_{7} \mathrm{H}_{6} \mathrm{O}$ & 106.1 & 956.4 & 311.712 & 1.15559 & 1.03 & 0.78 & 0.78 \\
\hline 13 & Heptanal-M & C111717 & $\mathrm{C}_{7} \mathrm{H}_{14} \mathrm{O}$ & 114.2 & 899.4 & 263.071 & 1.32928 & 2.38 & 3.03 & 2.45 \\
\hline 14 & Heptanal-D & C111717 & $\mathrm{C}_{7} \mathrm{H}_{14} \mathrm{O}$ & 114.2 & 899.4 & 263.071 & 1.70488 & 1.12 & 1.52 & 1.04 \\
\hline 15 & 2-Heptanone-M & C110430 & $\mathrm{C}_{7} \mathrm{H}_{14} \mathrm{O}$ & 114.2 & 892.3 & 256.991 & 1.26099 & 0.57 & 0.74 & 1.09 \\
\hline 16 & (E)-2-Hexenal & C6728263 & $\mathrm{C}_{6} \mathrm{H}_{10} \mathrm{O}$ & 98.1 & 846.5 & 232.455 & 1.18524 & 0.35 & 0.56 & 0.38 \\
\hline 17 & Butanoic acid & C107926 & $\mathrm{C}_{4} \mathrm{H}_{8} \mathrm{O}_{2}$ & 88.1 & 823.3 & 220.013 & 1.16356 & 0.60 & 0.36 & 0.32 \\
\hline 18 & Octanal-D & C124130 & $\mathrm{C}_{8} \mathrm{H}_{16} \mathrm{O}$ & 128.2 & 1004.1 & 356.912 & 1.83342 & 0.18 & 0.32 & 0.25 \\
\hline 19 & (E)-Hept-2-enal & C18829555 & $\mathrm{C}_{7} \mathrm{H}_{12} \mathrm{O}$ & 112.2 & 954.8 & 310.312 & 1.25847 & 0.18 & 0.45 & 0.31 \\
\hline 20 & Oct-1-en-3-ol & C3391864 & $\mathrm{C}_{8} \mathrm{H}_{16} \mathrm{O}$ & 128.2 & 982.1 & 333.612 & 1.16305 & 0.14 & 0.15 & 0.10 \\
\hline 21 & 3-Methylthiopropanal & C3268493 & $\mathrm{C}_{4} \mathrm{H}_{8} \mathrm{OS}$ & 104.2 & 904.4 & 267.364 & 1.09275 & 0.12 & 0.14 & 0.12 \\
\hline 22 & Cyclohexanone & C108941 & $\mathrm{C}_{6} \mathrm{H}_{10} \mathrm{O}$ & 98.1 & 897.4 & 261.358 & 1.15434 & 0.12 & 0.15 & 0.20 \\
\hline 23 & 2-Heptanone-D & C110430 & $\mathrm{C}_{7} \mathrm{H}_{14} \mathrm{O}$ & 114.2 & 890.1 & 255.781 & 1.63654 & 0.02 & 0.03 & 0.08 \\
\hline 24 & Isovaleric acid & C503742 & $\mathrm{C}_{5} \mathrm{H}_{10} \mathrm{O}_{2}$ & 102.1 & 856.8 & 237.978 & 1.22173 & 0.04 & 0.05 & 0.22 \\
\hline 25 & Furfural & C98011 & $\mathrm{C}_{5} \mathrm{H}_{4} \mathrm{O}_{2}$ & 96.1 & 828.8 & 222.963 & 1.08462 & 0.11 & 0.12 & 0.20 \\
\hline 26 & Hexanal-M & C66251 & $\mathrm{C}_{6} \mathrm{H}_{12} \mathrm{O}$ & 100.2 & 792.4 & 203.443 & 1.25543 & 3.53 & 4.16 & 3.57 \\
\hline 27 & Hexanal-D & C66251 & $\mathrm{C}_{6} \mathrm{H}_{12} \mathrm{O}$ & 100.2 & 792.4 & 203.443 & 1.56915 & 11.69 & 11.13 & 9.48 \\
\hline 28 & 2-Hexanone & C591786 & $\mathrm{C}_{6} \mathrm{H}_{12} \mathrm{O}$ & 100.2 & 782.3 & 198.296 & 1.18571 & 0.06 & 0.13 & 0.14 \\
\hline 29 & 2-Methylpropanoic acid & C79312 & $\mathrm{C}_{4} \mathrm{H}_{8} \mathrm{O}_{2}$ & 88.1 & 756.6 & 187.849 & 1.15816 & 0.06 & 0.08 & 0.51 \\
\hline 30 & Pentan-1-ol-M & C71410 & $\mathrm{C}_{5} \mathrm{H}_{12} \mathrm{O}$ & 88.1 & 762.4 & 190.202 & 1.24992 & 0.30 & 0.65 & 0.50 \\
\hline 31 & (E)-2-Pentenal-M & $\mathrm{C} 1576870$ & $\mathrm{C}_{5} \mathrm{H}_{8} \mathrm{O}$ & 84.1 & 747.5 & 184.159 & 1.1082 & 0.09 & 0.23 & 0.12 \\
\hline 32 & Pentanal-M & C110623 & $\mathrm{C}_{5} \mathrm{H}_{10} \mathrm{O}$ & 86.1 & 695.1 & 162.924 & 1.18389 & 1.75 & 2.48 & 2.13 \\
\hline 33 & Pentanal-D & C110623 & $\mathrm{C}_{5} \mathrm{H}_{10} \mathrm{O}$ & 86.1 & 693 & 162.061 & 1.42874 & 2.47 & 6.31 & 6.67 \\
\hline 34 & 2-Methylbutanal-M & C96173 & $\mathrm{C}_{5} \mathrm{H}_{10} \mathrm{O}$ & 86.1 & 665.1 & 153.946 & 1.16089 & 0.62 & 1.14 & 1.29 \\
\hline 35 & 2-Methylbutanal-D & C96173 & $\mathrm{C}_{5} \mathrm{H}_{10} \mathrm{O}$ & 86.1 & 663.2 & 153.428 & 1.40054 & 0.80 & 1.74 & 6.89 \\
\hline 36 & 3-Methylbutanal-M & C590863 & $\mathrm{C}_{5} \mathrm{H}_{10} \mathrm{O}$ & 86.1 & 645.9 & 148.767 & 1.1735 & 0.93 & 1.44 & 1.24 \\
\hline 37 & 3-Methylbutanal-D & C590863 & $\mathrm{C}_{5} \mathrm{H}_{10} \mathrm{O}$ & 86.1 & 645.9 & 148.767 & 1.41909 & 0.84 & 1.74 & 6.34 \\
\hline 38 & Methyl isobutyl ketone-M & C108101 & $\mathrm{C}_{6} \mathrm{H}_{12} \mathrm{O}$ & 100.2 & 730 & 177.081 & 1.18018 & 0.23 & 0.25 & 0.23 \\
\hline 39 & $n$-Propyl acetate & C109604 & $\mathrm{C}_{5} \mathrm{H}_{10} \mathrm{O}_{2}$ & 102.1 & 705.3 & 167.067 & 1.48439 & 0.14 & 0.04 & 0.05 \\
\hline 40 & (E)-2-pentenal-D & $\mathrm{C} 1576870$ & $\mathrm{C}_{5} \mathrm{H}_{8} \mathrm{O}$ & 84.1 & 746.2 & 183.641 & 1.36419 & 0.09 & 0.32 & 0.15 \\
\hline 41 & Pentan-1-ol-D & C71410 & $\mathrm{C}_{5} \mathrm{H}_{12} \mathrm{O}$ & 88.1 & 761.5 & 189.857 & 1.50813 & 0.07 & 0.21 & 0.12 \\
\hline 42 & Methyl isobutyl ketone & C108101 & $\mathrm{C}_{6} \mathrm{H}_{12} \mathrm{O}$ & 100.2 & 729.2 & 176.736 & 1.48587 & 0.14 & 0.05 & 0.06 \\
\hline 43 & Ethyl acetate-M & C141786 & $\mathrm{C}_{4} \mathrm{H}_{8} \mathrm{O}_{2}$ & 88.1 & 603 & 137.2 & 1.10078 & 2.34 & 2.77 & 2.71 \\
\hline 44 & Ethyl acetate-D & C141786 & $\mathrm{C}_{4} \mathrm{H}_{8} \mathrm{O}_{2}$ & 88.1 & 602.4 & 137.027 & 1.34341 & 22.92 & 15.92 & 12.18 \\
\hline 45 & 2-Butanone-D & C78933 & $\mathrm{C}_{4} \mathrm{H}_{8} \mathrm{O}$ & 72.1 & 573.5 & 129.258 & 1.25215 & 2.10 & 4.26 & 4.28 \\
\hline 46 & 2-Methylpropanal & C78842 & $\mathrm{C}_{4} \mathrm{H}_{8} \mathrm{O}$ & 72.1 & 552.7 & 123.628 & 1.2884 & 0.04 & 0.09 & 0.29 \\
\hline 47 & 2-Butanone-M & C78933 & $\mathrm{C}_{4} \mathrm{H}_{8} \mathrm{O}$ & 72.1 & 571.2 & 128.644 & 1.06223 & 0.74 & 0.86 & 0.79 \\
\hline 48 & 2,3-Butanedione & C431038 & $\mathrm{C}_{4} \mathrm{H}_{6} \mathrm{O}_{2}$ & 86.1 & 571.8 & 128.784 & 1.17135 & 1.13 & 1.16 & 0.94 \\
\hline 49 & Acetone & C67641 & $\mathrm{C}_{3} \mathrm{H}_{6} \mathrm{O}$ & 58.1 & 481.4 & 104.397 & 1.12439 & 11.78 & 14.00 & 12.82 \\
\hline 50 & Benzaldehyde-D & C100527 & $\mathrm{C}_{7} \mathrm{H}_{6} \mathrm{O}$ & 106.1 & 956.8 & 312.031 & 1.47659 & 0.09 & 0.06 & 0.06 \\
\hline
\end{tabular}

RI denotes the retention index calculated using $n$-ketones $\mathrm{C} 4-\mathrm{C} 9$ as foreign reference on MXT- 5 capillary chromatographic column. DT denotes the drift time in the IMS drift tube. RT represents retention time, MF molecular formulation, and MW molecular weight. - $\mathrm{M}$ and -D at the end of volatile chemicals denote monomer and dimer, respectively.

Rows from top to bottom were meatballs of BF, DFF, and HAF, respectively. Each column represents different samples of the same volatile substances (red signal, indicating the chemical concentration is relatively higher); the fingerprint has two of the same name which are the monomer and dimer
(Figure 4). The volatile flavor compounds of the samples before and after frying showed a certain difference (represented by red frame area in Figure 4). For volatile flavor substances in meatballs before frying, the relative contents of butyric acid, propyl acetate, benzaldehyde (including 


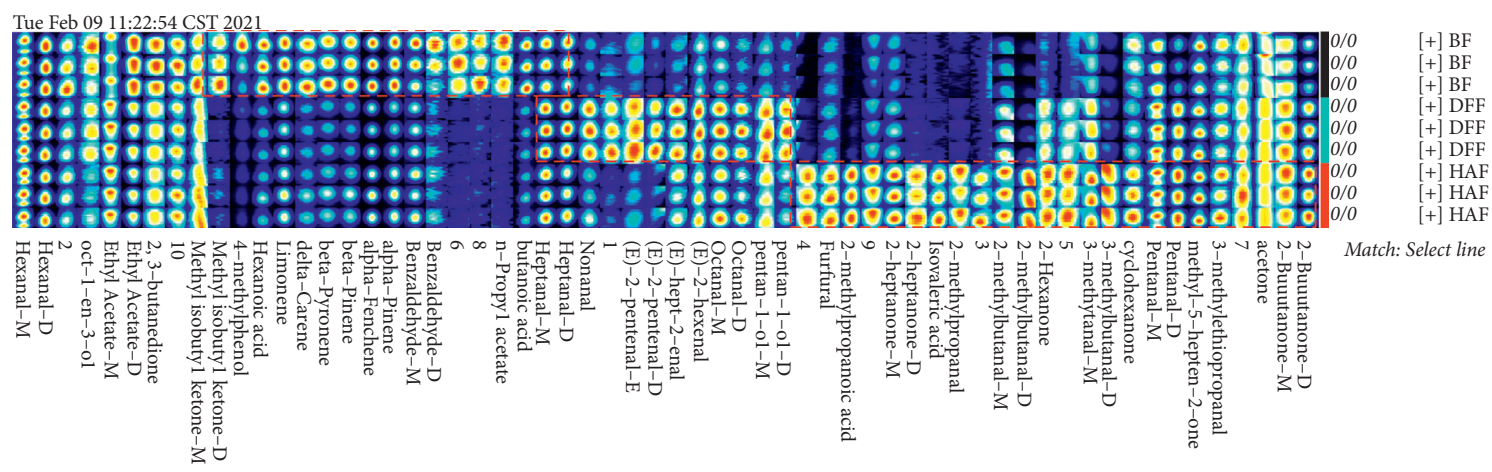

FIGURE 4: Fingerprint of volatile organic compounds of giant salamander meatball before and after frying.

monomer and dimer), dipentene, $\alpha$-pinene, $\beta$-pinene, 3 carene, and 4-methyl-2-pentanone (including monomer and dimer) were relatively high. For volatile components in meatballs by deep fat frying, the relative contents of 1pentanol (including monomer and dimer), n-octanal (including monomer and dimer), 2-hexenal, (E)-2-heptenal, nonanal, heptanal (including monomer and dimer), and (E)2-pentenal (including monomer and dimer) were relatively high. For volatile components in meatballs of hot air frying, the relative contents of acetone, 2-butanone (including monomer, dimer), 3-methyl sulfonium propanal, methyl heptyl ketene, amyl aldehyde (including monomer, dimer), cyclohexanone, isovaleric aldehyde (including monomer, dimer), 2-hexanone, 2-methyl butyl aldehyde (including monomer, dimer), butyl aldehyde, ketone of isovaleric acid, 2-heptyl (including monomer, dimer), isobutyric acid, and furfural were prominent. Figure 4 also shows that the kinds and contents of volatile components in HAF samples are more abundant than those in DFF samples, implying better flavor attributes.

The volatile flavor components during meatball frying are very complicated, generally containing aldehydes, alcohols, ketones, terpene derivatives, hydrocarbons, and a small amount of organic compounds such as furan, thioether, and naphthalene $[17,33]$. In order to better characterize the changes of various volatile compounds, the relative content changes of volatile components in giant salamander meatballs with different frying methods were obtained according to the signal intensity of the compounds on the fingerprint, shown in Figure 5. The volatile components before frying are mainly aldehydes, ketones, esters, and alkenes, followed by acids, alcohols, and phenols. The relative contents of aldehydes, ketones, esters, alkenes, acids, alcohols, and phenols were $30.25-46.40 \%$, $19.94-25.30 \%, 14.94-25.39 \%, 9.40-16.05 \%, 1.87-3.43 \%$, $0.51-1.02 \%$, and $1.00-4.42 \%$, respectively. Previous reports showed that aldehydes were mainly the products of lipid oxidation, and the threshold value was low, which contributed to the volatile flavor of minced meat products. Ketones and alcohols also come from oxidative degradation of fatty acids, and their thresholds are higher than aldehydes, with pleasant flavors such as floral scent and fruity aroma. Esters are mainly the products of esterification of acids and alcohols [33-35].

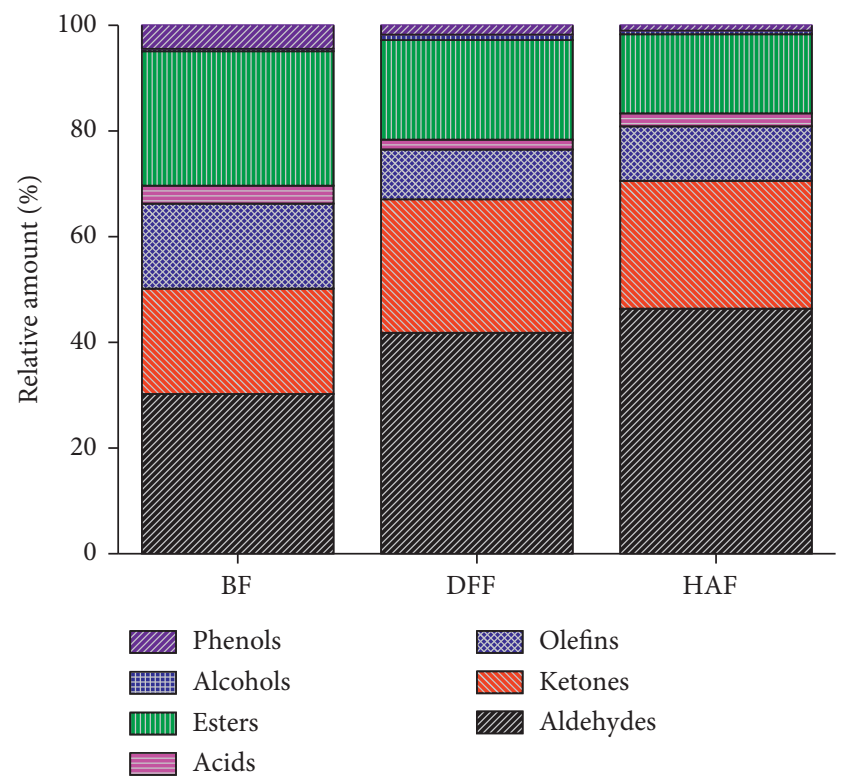

FIGURE 5: Changes of volatile flavor components of giant salamander meatball before and after frying.

Figure 5 also shows that, before frying, the volatile organic compounds of giant salamander meatballs contained aldehydes (30.25\%), ketones (19.94\%), esters (25.39\%), and alkenes (16.05\%). After deep fat frying and hot air frying, the relative amount of aldehydes increased by $37.85 \%$ and $53.38 \%$, respectively, while the relative content of ketones increased by $26.88 \%$ and $21.21 \%$, respectively. The relative content of esters decreased by $25.79 \%$ and $41.16 \%$, respectively, while the relative content of olefins decreased by $41.37 \%$ and $35.76 \%$, respectively. The relative changes of acids, phenols, and alcohols were not obvious before and after frying. During frying process of meat products, the proportion of aldehydes and ketones increased due to the intensifying of lipid oxidative degradation, which had an important impact on the flavor of minced meat products; especially ketones contributed to reducing the fishy smell of aquatic products $[17,33]$. The present study showed that, after frying, the relative contents of aldehydes and ketones for giant salamander meatballs treated by deep fat frying and hot air frying samples increased significantly, whereas the relative contents of esters and olefins decreased obviously, 


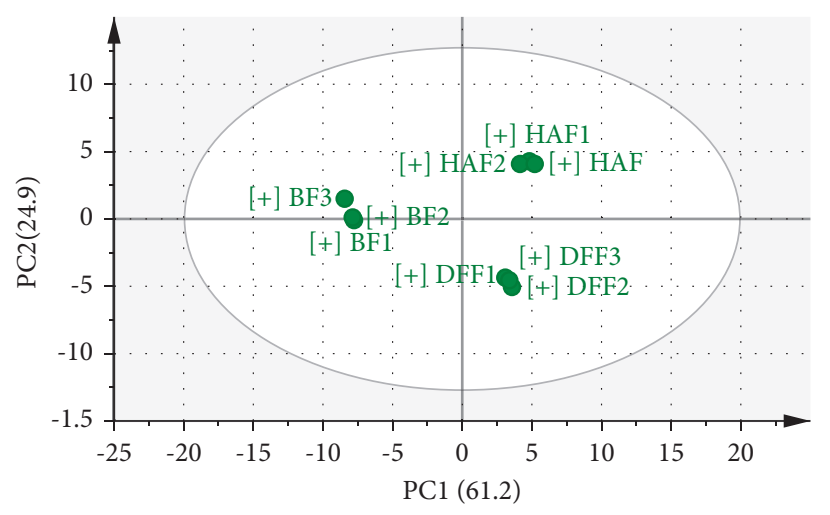

(a)

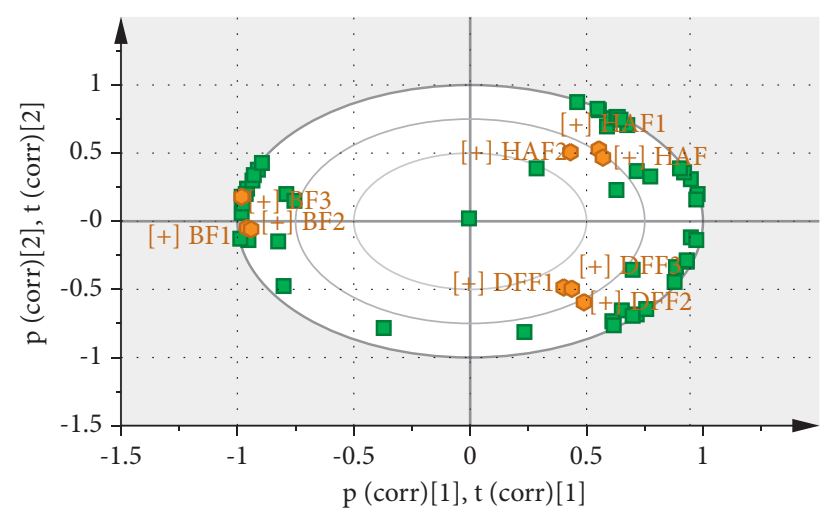

(b)

FIGURE 6: PCA score and biplot diagram of volatile flavor compounds of giant salamander meatball before and after two frying methods. (a) Score plot; (b) biplot diagram.

and the increase or decrease of hot air frying samples was slightly larger than that of deep fat frying, which may cause differences in quality and flavor characteristics of final products.

\subsection{Principal Component Analysis of Giant Salamander} Meatballs before and after Frying. Principal component analysis (PCA) is an important method of data dimensionality reduction. Previous studies have shown that GCIMS spectrum data combined with PCA can be used to distinguish the origin, different parts, and cooking methods of food raw materials according to volatile flavor substances [36-39]. The GC-IMS chromatographic data of samples before and after frying were used. According to volatile flavor fingerprint of samples of different fried giant salamander meatballs, the score plot and biplot diagram of principal component analysis results are shown in Figures 6(a) and 6(b).

The cumulative contribution rate of the first two principal components reached $86.1 \%$, which could represent the majority of information of the meatball samples (Figures 6(a) and 6(b)). The data of flavor substance spectrum of the same group of giant salamander meatballs gathered together, which made the samples of different frying methods better distinguish before and after frying, and there is no obvious overlap area. This result shows that GC-IMS technology together with PCA can realize better identification of volatile components of giant salamander meatballs before and after frying, and between deep fat frying and hot air frying. The results are similar to GC-IMS and PCA reports on volatile flavor substances of different parts of giant salamander meat, different places of smoked chicken, and squid cooking methods [11, 36-39].

\section{Conclusion}

In summary, compared with deep fat frying, hot air-fried giant salamander meatballs had higher hardness, elasticity, and $L^{*}(p<0.05)$, but the $a^{*}, b^{*}$, fat content, and yield were relatively low $(p<0.05)$. There were no significant differences in sensory score, cohesiveness, and chewiness between the two frying methods $(p<0.05)$. Fifty volatile flavor compounds, including 22 aldehydes, 11 ketones, 6 alkenes, 4 acids, 3 esters, 3 alcohols, and 1 phenolic, were identified from giant salamander meatballs by HS-GC-IMS technique. Compared with the unfried meatballs, the relative contents of aldehydes and ketones increased significantly, while the relative contents of esters and alkenes decreased significantly, and the increase or decrease of these main volatile substances in meatballs by hot air frying was slightly larger than that of deep fat frying. The volatile flavor compounds before and after frying, and between deep fat frying and hot air frying, could be well distinguished through GC-IMS signals combined with principal component analysis. This study provided a reference for the development and quality control of precooked giant salamander meatballs in future.

\section{Data Availability}

All data generated or analyzed during this study are included in this article.

\section{Conflicts of Interest}

The authors declare that there are no conflicts of interest regarding the publication of this study.

\section{Authors' Contributions}

Wengang Jin and Jinjin Pei contributed equally to this work. Wengang Jin and Jinjin Pei conducted investigation, wrote the original draft, and performed plot analysis. Xiaohua Chen, Jingzhang Geng, and Dejing Chen performed partial analysis, visualization, and language check. Jinjin Pei and Ruichang Gao reviewed and edited the manuscript, supervised the work, and acquired funding.

\section{Acknowledgments}

This investigation was funded by Collaborative Innovation Center Project for Qin-Ba Mountain Bio-Resources 
Utilization and Development (no. QBXT-18-04), National Natural Science Foundation of China (no. 31801563), and China Scholarship Council (no. 202008610071). The authors also appreciate the partial fund from Shaanxi "Sanqin Scholar" Innovation Team and Hanzhong Youth Innovation Team for giant salamander processing and utilization.

\section{References}

[1] J. Pei, D. Chen, W. Jin et al., "Structure and mode of action of a novel antibacterial peptide from the blood of Andrias davidianus," Letters in Applied Microbiology, vol. 69, no. 5, pp. 312-317, 2019.

[2] D. He, W. Zhu, W. Zeng et al., "Nutritional and medicinal characteristics of Chinese giant salamander (Andrias davidianus) for applications in healthcare industry by artificial cultivation: a review," Food Science and Human Wellness, vol. 7, no. 1, pp. 1-10, 2018.

[3] C. Lu, J. Chai, R. W. Murphy, and J. Che, "Giant salamanders: farmed yet endangered," Science, vol. 367, no. 6481, 989 pages, 2020.

[4] W.-G. Jin, J. Pei, Y.-N. Du et al., "Characterization and functional properties of gelatin extracted from Chinese giant salamander (Andrias davidianus) skin," Journal of Aquatic Food Product Technology, vol. 28, no. 8, pp. 861-876, 2019.

[5] D. Ni, Z. He, D. Zhang, and D. Deng, "Changes in nutritional quality during processing of giant salamander soup," Food Science, vol. 38, no. 20, pp. 119-224, 2017.

[6] Y. F. Hu, N. N. Li, J. R. Chen, Y. S. Jia, J. Liang, and Y. F. Li, "Effect of chlorine dioxide on quality of giant salamander cutting meats in small modified atmosphere packaging," Advance Journal of Food Science and Technology, vol. 10, no. 4, pp. 302-308, 2016.

[7] A. H. Ramadhan, T. Nawas, X. W. Zhang, W. M. Pembe, W. S. Xia, and Y. S. Xu, "Purification and identification of a novel antidiabetic peptide from Chinese giant salamander (Andrias davidianus) protein hydrolysate against $\alpha$-amylase and $\alpha$-glucosidase," International Journal of Food Properties, vol. 20, pp. s3360-s3372, 2018.

[8] J. Pei and L. Jiang, "Antimicrobial peptide from mucus of Andrias davidianus: screening and purification by magnetic cell membrane separation technique," International Journal of Antimicrobial Agents, vol. 50, no. 1, pp. 41-46, 2017.

[9] W. Zhu, Y. Ji, Y. Wang et al., "Structural characterization and in vitro antioxidant activities of chondroitin sulfate purified from Andrias davidianus cartilage," Carbohydrate Polymers, vol. 196, pp. 398-404, 2018.

[10] J. Pan, H. Lian, M. Shang et al., "Physicochemical properties of Chinese giant salamander (Andrias davidianus) skin gelatin as affected by extraction temperature and in comparison with fish and bovine gelatin," Journal of Food Measurement and Characterization, vol. 14, no. 5, pp. 2656-2666, 2020.

[11] W. Jin, P. Zhao, J. Jin et al., "Volatile component fingerprint analysis of giant salamander (Andrias davidiauns) from different edible parts based on gas chromatography-ion mobility spectroscopy," Food Science, 2021.

[12] S. A. Rubel, Z. N. Yu, H. M. Murshed et al., "Addition of olive (olea europaea) leaf extract as a source of natural antioxidant in mutton meatball stored at refrigeration temperature," Journal of Food Science \& Technology, vol. 58, pp. 4002-4010, 2021.

[13] H. S. Cheon, S.-H. Choi, C. Jhin et al., "Optimization of sterilization conditions for production of retorted meatballs,"
Food Science and Biotechnology, vol. 24, no. 2, pp. 471-480, 2015.

[14] Y. Li, H. Li, X. He, and Y. Wei, "Effect of day lily powder addition on the quality of beef meatballs based on fuzzy mathematics sensory evaluation methodology," Food and Fermentation Industries, vol. 47, no. 5, pp. 112-119, 2021.

[15] P. Ateba and G. S. Mittal, "Dynamics of crust formation and kinetics of quality changes during frying of meatballs," Journal of Food Science, vol. 59, no. 6, pp. 1275-1278, 1994.

[16] Y. Wang, W. Zhang, and G. Zhou, "Effects of ultrasoundassisted frying on the physiochemical properties and microstructure of fried meatballs," International Journal of Food Science and Technology, vol. 54, no. 10, pp. 2915-2926, 2019.

[17] J. Zhang, Y. Zhang, Y. Wang, L. Xing, and W. Zhang, "Influences of ultrasonic-assisted frying on the flavor characteristics of fried meatballs," Innovative Food Science \& Emerging Technologies, vol. 62, Article ID 102365, 2020.

[18] L. N. F. Aslinah, M. Mat Yusoff, and M. R. Ismail-Fitry, "Simultaneous use of adzuki beans (Vigna angularis) flour as meat extender and fat replacer in reduced-fat beef meatballs (bebola daging)," Journal of Food Science \& Technology, vol. 55, no. 8, pp. 3241-3248, 2018.

[19] W. Zhao, M. Zhao, K. Wang et al., "Effect of frying methods on the eating quality of Coregonus peled meat," Food Science, vol. 41, no. 2, pp. 72-79, 2021.

[20] X. Yu, L. Li, J. Xue et al., "Effect of air-frying conditions on the quality attributes and lipidomic characteristics of surimi during processing," Innovative Food Science \& Emerging Technologies, vol. 60, Article ID 102305, 2020.

[21] A. N. Zaghi, S. M. Barbalho, E. L. Guiguer, and A. M. Otoboni, "Frying process: from conventional to air frying technology," Food Reviews International, vol. 35, no. 8, pp. 763-777, 2019.

[22] M. Zhang, G. Song, H. Wang, W. Rao, H. Wang, and Q. Shen, "Real-time detection of lipidomic characteristics of air-fried Hairtail by iKnife rapid evaporative ionization mass spectrometry," Food Science, vol. 41, no. 14, pp. 314-320, 2020.

[23] G. Song, M. Zhang, X. Yu et al., "Rapid detection the lipidomics profile of white shrimp (Penaeus vannamei) during air frying processing," Journal of Nuclear Agricultural Sciences, vol. 34, no. 6, pp. 1237-1247, 2020.

[24] A. Ghaitaranpour, A. Koocheki, M. Mohebbi, and M. O. Ngadi, "Effect of deep fat and hot air frying on doughnuts physical properties and kinetic of crust formation," Journal of Cereal Science, vol. 83, pp. 25-31, 2018.

[25] A. Ghaitaranpour, M. Mohebbi, A. Koocheki, and M. O. Ngadi, "An agent-based coupled heat and water transfer model for air frying of doughnut as a heterogeneous multiscale porous material," Innovative Food Science \& Emerging Technologies, vol. 61, Article ID 102335, 2020.

[26] T. Gouyo, C. Mestres, I. Maraval, B. Fontez, C. Hofleitner, and P. Bohuon, "Assessment of acoustic-mechanical measurements for texture of French fries: comparison of deep-fat frying and air frying," Food Research International, vol. 131, Article ID 108947, 2020.

[27] T.-K. Kim, H.-W. Kim, Y.-Y. Lee, H. W. Jang, Y.-B. Kim, and Y.-S. Choi, "Quality characteristics of duck jerky: combined effects of collagen and konjac," Poultry Science, vol. 99, no. 1, pp. 629-636, 2020.

[28] W. Jin, X. Chen, J. Geng et al., "Quality characteristics and moisture mobility of giant salamander (Andrias davidianus) jerky during roasting process," Journal of Food Quality, vol. 2021, Article ID 9970797, 11 pages, 2021.

[29] W. Jin, J. Pei, S. Wang, X. Chen, R. Gao, and M. Tan, "Effect of continuous and intermittent drying on water mobility of fresh 
walnuts (Juglans regia L.): a LF-NMR study," Drying Technology, pp. 1-11, 2020.

[30] M. Li, R. Yang, H. Zhang, S. Wang, D. Chen, and S. Lin, "Development of a flavor fingerprint by HS-GC-IMS with PCA for volatile compounds of Tricholoma matsutake Singer," Food Chemistry, vol. 290, pp. 32-39, 2019.

[31] S. Wang, H. Chen, and B. Sun, "Recent progress in food flavor analysis using gas chromatography-ion mobility spectrometry (GC-IMS)," Food Chemistry, vol. 315, Article ID 126158, 2020.

[32] X. Yang, K. Zhu, H. Guo et al., "Characterization of volatile compounds in differently coloured Chenopodium quinoa seeds before and after cooking by headspace-gas chromatography-ion mobility spectrometry," Food Chemistry, vol. 348, Article ID 129086, 2021.

[33] Z. Cui, H. Yan, T. Manoli, H. Mo, H. Li, and H. Zhang, "Changes in the volatile components of squid (illex argentinus) for different cooking methods via headspace-gas chromatography-ion mobility spectrometry," Food Sciences and Nutrition, vol. 8, no. 10, pp. 5748-5762, 2020.

[34] W. Yao, Y. Cai, D. Liu et al., "Comparative analysis of characteristic volatile compounds in Chinese traditional smoked chicken (specialty poultry products) from different regions by headspace-gas chromatography-ion mobility spectrometry," Poultry Science, vol. 99, no. 12, pp. 7192-7201, 2020.

[35] D. Feng, J. Wang, X.-J. Ji, W.-X. Min, and W.-J. Yan, “Analysis of volatile organic compounds by HS-GC-IMS in powdered yak milk processed under different sterilization conditions," Journal of Food Quality, vol. 2021, Article ID 5536645, 10 pages, 2021.

[36] L. Chang, S. Lin, B. Zou, X. Zheng, S. Zhang, and Y. Tang, "Effect of frying conditions on self-heating fried Spanish mackerel quality attributes and flavor characteristics," Foods, vol. 10, no. 1, 98 pages, 2021.

[37] X. Li, K. Wang, R. Yang, Y. Dong, and S. Lin, "Mechanism of aroma compounds changes from sea cucumber peptide powders (SCPPs) under different storage conditions," Food Research International, vol. 128, Article ID 108757, 2020.

[38] J. Song, Y. Shao, Y. Yan, X. Li, J. Peng, and L. Guo, "Characterization of volatile profiles of three colored quinoas based on GC-IMS and PCA," Lebensmittel-Wissenschaft \& Technologie, vol. 146, Article ID 111292, 2021.

[39] X. Fan, X. Jiao, J. Liu, M. Jia, C. Blanchard, and Z. Zhou, "Characterizing the volatile compounds of different sorghum cultivars by both GC-MS and HS-GC-IMS," Food Research International, vol. 140, Article ID 109975, 2021. 